Bull. Soc. math. France

132 (1), 2004, p. $137-156$

\title{
ON SQUARE FUNCTIONS ASSOCIATED TO SECTORIAL OPERATORS
}

\author{
By Christian Le Merdy
}

Dedicated to Alan McIntosh on the occasion of his 60th birthday ABStRACT. - We give new results on square functions

$$
\|x\|_{F}=\left\|\left(\int_{0}^{\infty}|F(t A) x|^{2} \frac{\mathrm{d} t}{t}\right)^{1 / 2}\right\|_{p}
$$

associated to a sectorial operator $A$ on $L^{p}$ for $1<p<\infty$. Under the assumption that $A$ is actually $R$-sectorial, we prove equivalences of the form $K^{-1}\|x\|_{G} \leq\|x\|_{F} \leq K\|x\|_{G}$ for suitable functions $F, G$. We also show that $A$ has a bounded $H^{\infty}$ functional calculus with respect to $\|\cdot\|_{F}$. Then we apply our results to the study of conditions under which we have an estimate $\left\|\left(\int_{0}^{\infty}\left|C \mathrm{e}^{-t A}(x)\right|^{2} \mathrm{~d} t\right)^{1 / 2}\right\|_{q} \leq M\|x\|_{p}$, when $-A$ generates a bounded semigroup $\mathrm{e}^{-t A}$ on $L^{p}$ and $C: D(A) \rightarrow L^{q}$ is a linear mapping.

RÉSUMÉ (Sur les fonctions carrées associées aux opérateurs sectoriels)

Nous obtenons de nouveaux résultats sur les fonctions carrées

$$
\|x\|_{F}=\left\|\left(\int_{0}^{\infty}|F(t A) x|^{2} \frac{\mathrm{d} t}{t}\right)^{1 / 2}\right\|_{p}
$$

associées à un opérateur sectoriel $A$ sur $L^{p}$ pour $1<p<\infty$. Quand $A$ est en fait $R$-sectoriel, on montre des équivalences de la forme $K^{-1}\|x\|_{G} \leq\|x\|_{F} \leq K\|x\|_{G}$ pour des fonctions $F, G$ appropriées. On démontre également que $A$ possède un calcul fonctionnel $H^{\infty}$ borné par rapport à $\|\cdot\|_{F}$. Puis nous appliquons nos résultats à l'étude de conditions impliquant une inégalité du type $\left\|\left(\int_{0}^{\infty}\left|C \mathrm{e}^{-t A}(x)\right|^{2} \mathrm{~d} t\right)^{1 / 2}\right\|_{q} \leq M\|x\|_{p}$ où $-A$ engendre un semigroupe borné $\mathrm{e}^{-t A}$ sur $L^{p}$ et $C: D(A) \rightarrow L^{q}$ est une application linéaire.

Texte reçu le 3 juillet 2002, accepté le 30 janvier 2003

Christian Le Merdy, Département de Mathématiques, Université de Franche-Comté, 25030 Besançon Cedex (France) • E-mail : lemerdy@math.univ-fcomte.fr 2000 Mathematics Subject Classification. - 47A60, 47D06.

Key words and phrases. - Sectorial operators, $H^{\infty}$ functional calculus, square functions, $R$-boundedness, admissibility. 


\section{Introduction}

The main objects of this paper will be bounded analytic semigroups and sectorial operators on $L^{p}$-spaces, their $H^{\infty}$ functional calculus, and their associated square functions. This beautiful and powerful subject grew out of McIntosh's seminal paper [18] and subsequent important works by McIntoshYagi [19] and Cowling-Doust-McIntosh-Yagi [6].

We first briefly recall a few classical notions which are the starting point of the whole theory. Given a Banach space $X$, we will denote by $B(X)$ the Banach algebra of all bounded operators on $X$. For any $\omega \in(0, \pi)$, we let

$$
\Sigma_{\omega}=\left\{z \in \mathbb{C}^{*} ;|\operatorname{Arg}(z)|<\omega\right\}
$$

be the open sector of angle $2 \omega$ around the half-line $(0, \infty)$. Let $A$ be a possibly unbounded operator $A$ on $X$ and assume that $A$ is closed and densely defined. For any $z$ in the resolvent set of $A$ we let $R(z, A)=(z-A)^{-1}$ denote the corresponding resolvent operator. Let $\sigma(A)$ denote the spectrum of $A$. Then by definition, $A$ is sectorial of type $\omega$ if the following three conditions are fulfilled:

(S1) $\sigma(A) \subset \bar{\Sigma}_{\omega}$.

(S2) For any $\theta \in(\omega, \pi)$ there is a constant $K_{\theta}>0$ such that

$$
\|z R(z, A)\| \leq K_{\theta}, \quad z \in \bar{\Sigma}_{\theta}^{c} .
$$

(S3) $A$ has a dense range.

Very often, (S3) is unnecessary and omitted in the definition of sectoriality. However we include it here to avoid tedious technical discussions. Note the well-known fact that $A$ is one-to-one if it satisfies (S1), (S2) and (S3) above.

Given any $\theta \in(0, \pi)$, we let $H^{\infty}\left(\Sigma_{\theta}\right)$ be the algebra of all bounded analytic functions $f: \Sigma_{\theta} \rightarrow \mathbb{C}$ and we let $H_{0}^{\infty}\left(\Sigma_{\theta}\right)$ be the subalgebra of all $f \in H^{\infty}\left(\Sigma_{\theta}\right)$ for which there exist two positive numbers $s, c>0$ such that

$$
|f(z)| \leq c \frac{|z|^{s}}{(1+|z|)^{2 s}}, \quad z \in \Sigma_{\theta}
$$

Now given a sectorial operator $A$ of type $\omega \in(0, \pi)$ on a Banach space $X$, a number $\theta \in(\omega, \pi)$, and a function $f \in H_{0}^{\infty}\left(\Sigma_{\theta}\right)$, one may define an operator $f(A) \in B(X)$ as follows. We let $\gamma \in(\omega, \theta)$ be an intermediate angle and consider the oriented contour $\Gamma_{\gamma}$ defined by

$$
\Gamma_{\gamma}(t)=\left\{\begin{array}{cc}
-t \mathrm{e}^{i \gamma} & t \in \mathbb{R}_{-}, \\
t \mathrm{e}^{-i \gamma} & t \in \mathbb{R}_{+}
\end{array}\right.
$$

Then we let

$$
f(A)=\frac{1}{2 \pi i} \int_{\Gamma_{\gamma}} f(z) R(z, A) \mathrm{d} z .
$$

TOME $132-2004-\mathrm{N}^{\mathrm{O}} 1$ 
It follows from Cauchy's Theorem that the definition of $f(A)$ does not depend on the choice of $\gamma$ and it can be shown that the mapping $f \mapsto f(A)$ is an algebra homomorphism from $H_{0}^{\infty}\left(\Sigma_{\theta}\right)$ into $B(X)$. The next step in $H^{\infty}$ functional calculus consists in the definition of a possibly unbounded operator $f(A)$ associated to any $f \in H^{\infty}\left(\Sigma_{\theta}\right)$. Since we shall not use this construction here, we omit it and refer the reader to [18], [19] and [6] for details. We merely recall that by definition, $A$ admits a bounded $H^{\infty}\left(\Sigma_{\theta}\right)$ functional calculus if $f(A)$ is bounded for any $f \in H^{\infty}\left(\Sigma_{\theta}\right)$. In that case, the mapping $f \mapsto f(A)$ is a bounded homomorphism from $H^{\infty}\left(\Sigma_{\theta}\right)$ into $B(X)$, provided that $H^{\infty}\left(\Sigma_{\theta}\right)$ is equipped with the norm

$$
\|f\|_{\infty, \theta}=\sup \left\{|f(z)| ; z \in \Sigma_{\theta}\right\} .
$$

We shall be mainly concerned by square functions associated to sectorial operators in the case when $X$ is an $L^{p}$-space. For any $\omega \in(0, \pi)$, we introduce

$$
H_{0}^{\infty}\left(\Sigma_{\omega+}\right)=\bigcup_{\theta>\omega} H_{0}^{\infty}\left(\Sigma_{\theta}\right)
$$

Assume first that $X=H$ is a Hilbert space. Given a sectorial operator $A$ of type $\omega$ on $H$ and $F \in H_{0}^{\infty}\left(\Sigma_{\omega+}\right)$, we consider

$$
\|x\|_{F}=\left(\int_{0}^{\infty}\|F(t A) x\|^{2} \frac{\mathrm{d} t}{t}\right)^{1 / 2}, \quad x \in H,
$$

which may be either finite or infinite. These square function norms were introduced in [18] where it is shown that for any $\theta>\omega$ and any non zero $F \in H_{0}^{\infty}\left(\Sigma_{\omega+}\right), A$ has a bounded $H^{\infty}\left(\Sigma_{\theta}\right)$ functional calculus if and only if $\left\|_{.}\right\|_{F}$ is equivalent to the original norm of $H$. In [19, Theorem 5], McIntosh-Yagi established the following two remarkable properties. First these square function norms are pairwise equivalent, that is, for any two non zero functions $F$ and $G$ in $H_{0}^{\infty}\left(\Sigma_{\omega+}\right)$ there exists a constant $K>0$ such that $K^{-1}\|x\|_{G} \leq\|x\|_{F} \leq K\|x\|_{G}$ for any $x \in H$. Second, $A$ always has a bounded $H^{\infty}$ functional calculus with respect to \|\|$_{F}$. More precisely, for any $\theta>\omega$ and for any $F \in H_{0}^{\infty}\left(\Sigma_{\theta}\right)$, there is a constant $K>0$ such that $\|f(A) x\|_{F} \leq K\|f\|_{\infty, \theta}\|x\|_{F}$ for any $f \in H^{\infty}\left(\Sigma_{\theta}\right)$ and any $x \in H$. Further properties and applications of square functions $\|\cdot\|_{F}$ were investigated in [3], to which we refer the interested reader.

We now turn to $L^{p}$-spaces. Let $1 \leq p<\infty$ be a number, let $\Omega$ be an arbitrary measure space, and consider the Banach space $X=L^{p}(\Omega)$. Given a sectorial operator $A$ of type $\omega$ on $L^{p}(\Omega)$ and $F \in H_{0}^{\infty}\left(\Sigma_{\omega+}\right)$, we let

$$
\|x\|_{F}=\left\|\left(\int_{0}^{\infty}|F(t A) x|^{2} \frac{\mathrm{d} t}{t}\right)^{1 / 2}\right\|_{L^{p}(\Omega)}, \quad x \in L^{p}(\Omega) .
$$

Again $\|x\|_{F}$ may be either finite or infinite. These square function norms were introduced in [6] and play a key role in the study of bounded $H^{\infty}$ functional calculus on $L^{p}$-spaces (see Corollary 2.3 below). The latter definition obviously extends the previous one that we recover when $p=2$. However it is unknown 
whether the results from [19] reviewed above extend to the case when $p \neq 2$. In particular it is unknown whether square function norms are pairwise equivalent on $L^{p}$-spaces. In a recent work [2], Auscher-Duong-McIntosh succeded in proving such an equivalence in the case when $-A$ generates a bounded analytic semigroup acting on $L^{2}(\Omega)$ with suitable upper bounds on its heat kernels. We shall prove that the results from [19, Theorem 5$]$ actually extend to all operators which are not only sectorial but $R$-sectorial. This notion which arose from some recent work of Weis [22] will be explained at the beginning of the next section.

Theorem 1.1. - Let $A$ be an $R$-sectorial operator of $R$-type $\omega \in(0, \pi)$ on a space $L^{p}(\Omega)$, with $1 \leq p<\infty$. Let $\theta \in(\omega, \pi)$ and let $F$ and $G$ be two non zero functions belonging to $H_{0}^{\infty}\left(\Sigma_{\theta}\right)$.

1) There exists a constant $K>0$ such that for any $f \in H^{\infty}\left(\Sigma_{\theta}\right)$ and any $x \in L^{p}(\Omega)$, we have

$$
\begin{aligned}
\|\left(\int_{0}^{\infty}|f(A) F(t A) x|^{2} \frac{\mathrm{d} t}{t}\right)^{1 / 2} & \|_{L^{p}(\Omega)} \\
& \leq K\|f\|_{\infty, \theta}\left\|\left(\int_{0}^{\infty}|G(t A) x|^{2} \frac{\mathrm{d} t}{t}\right)^{1 / 2}\right\|_{L^{p}(\Omega)} .
\end{aligned}
$$

2) There exists a constant $K>0$ such that

$$
K^{-1}\|x\|_{G} \leq\|x\|_{F} \leq K\|x\|_{G}, \quad x \in L^{p}(\Omega) .
$$

This result will be proved in Section 2 below, where we also include some relevant comments. Then Section 3 is devoted to an application of Theorem 1.1 to the study of $R$-admissibility. This new concept is a natural extension of the classical notion of admissibility considered e.g. in [24], [23], [25], [8] or [16]. Given a bounded analytic semigroup $T_{t}=\mathrm{e}^{-t A}$ on $L^{p}(\Omega)$ and a linear mapping $C$ from the domain of $A$ into some $L^{q}(\Sigma)$, we will study conditions under which we have an estimate of the form

$$
\left\|\left(\int_{0}^{\infty}\left|C T_{t}(x)\right|^{2} \mathrm{~d} t\right)^{1 / 2}\right\|_{L^{q}(\Sigma)} \leq M\|x\|_{L^{p}(\Omega)} .
$$

In particular we will show that such an estimate holds if $A$ has a bounded $H^{\infty}\left(\Sigma_{\theta}\right)$ functional calculus for some $\theta<\frac{1}{2} \pi$ and the set $\left\{(-s)^{1 / 2} C R(s, A) ; s \in\right.$ $\mathbb{R}, s<0\}$ is $R$-bounded. This extends a result of ours ([16]) corresponding to the case when $p=2$.

Acknowledgements. - This research was carried out while I was visiting the Centre for Mathematics and its Applications at the Australian National University in Canberra. It is a pleasure to thank the CMA for its warm hospitality. I am also grateful to Pascal Auscher, Xuan Thinh Duong, and Alan McIntosh for having informed me of [2] and for stimulating discussions on these topics.

TOME $132-2004-\mathrm{N}^{\mathrm{O}} 1$ 


\section{Equivalence of square function norms}

The main purpose of this section is the proof of Theorem 1.1. We first recall the key concepts of $R$-boundedness (see [4]) and $R$-sectoriality (see [22], [21], [14]). Consider a Rademacher sequence $\left(\varepsilon_{k}\right)_{k \geq 1}$ on a probability space $\left(\Omega_{0}, \mathbb{P}\right)$. That is, the $\varepsilon_{k}$ 's are pairwise independent random variables on $\Omega_{0}$ and $\mathbb{P}\left(\varepsilon_{k}=1\right)=\mathbb{P}\left(\varepsilon_{k}=-1\right)=\frac{1}{2}$ for any $k \geq 1$. Then for any finite family $x_{1}, \ldots, x_{n}$ in a Banach space $X$, we let

$$
\left\|\sum_{k=1}^{n} \varepsilon_{k} x_{k}\right\|_{\operatorname{Rad}(X)}=\int_{\Omega_{0}}\left\|\sum_{k=1}^{n} \varepsilon_{k}(s) x_{k}\right\|_{X} \mathrm{~d} \mathbb{P}(s) .
$$

Let $X, Y$ be two Banach spaces and let $B(X, Y)$ denote the space of all bounded operators from $X$ into $Y$. By definition, a set $\mathcal{T} \subset B(X, Y)$ is $R$-bounded if there is a constant $C \geq 0$ such that for any finite families $T_{1}, \ldots, T_{n}$ in $\mathcal{T}$, and $x_{1}, \ldots, x_{n}$ in $X$, we have

$$
\left\|\sum_{k=1}^{n} \varepsilon_{k} T_{k}\left(x_{k}\right)\right\|_{\operatorname{Rad}(Y)} \leq C\left\|\sum_{k=1}^{n} \varepsilon_{k} x_{k}\right\|_{\operatorname{Rad}(X)} .
$$

In that case, the smallest possible $C$ is called the $R$-boundedness constant of $\mathcal{T}$ and is denoted by $R(\mathcal{T})$. If $A$ is a sectorial operator on $X$ and $\omega \in(0, \pi)$ is a number, we say that $A$ is $R$-sectorial of $R$-type $\omega$ if for any $\theta \in(\omega, \pi)$, the set $\left\{z R(z, A) ; z \in \bar{\Sigma}_{\theta}^{c}\right\} \subset B(X)$ is $R$-bounded.

To describe the range of applications of our result, we first recall that if $X$ is a Hilbert space, then any bounded subset of $B(X)$ is $R$-bounded, hence any sectorial operator of type $\omega$ on $X$ is actually $R$-sectorial of $R$-type $\omega$. Thus Theorem 1.1 comprises [19, Theorem 5] that we recover when $p=2$. Note that our proof reduces to that of [19] in this case. If $X$ is not isomorphic to a Hilbert space, then there exist bounded subsets of $B(X)$ which are not $R$ bounded (see e.g. [1, Proposition 1.13]). The notion of $R$-sectoriality on non Hilbertian Banach spaces is closely related to maximal $L^{p}$-regularity. Namely, it was proved in [13] and [22] that if $A$ is a sectorial operator of type $<\frac{1}{2} \pi$ on a Banach space $X$ with maximal $L^{p}$-regularity, then $A$ is $R$-sectorial of $R$-type $<\frac{1}{2} \pi$. Thus the counterexamples to maximal $L^{p}$-regularity obtained by Kalton-Lancien [13] show that when $p \neq 2$, there exist sectorial operators on $L^{p}$-spaces which are not $R$-sectorial. Conversely, it was proved in [22] that if $X$ is a UMD Banach space, and $A$ is $R$-sectorial of $R$-type $<\frac{1}{2} \pi$ on $X$, then $A$ has maximal $L^{p}$-regularity. Thus for $1<p<\infty$ and $\omega<\frac{1}{2} \pi$, Theorem 1.1 exactly applies when the operator $A$ has maximal $L^{p}$-regularity. In particular it applies to the operators considered in [2].

BUlletin DE LA SOCiÉtÉ MATHÉmATiQUe DE FRANCE 
If $X=L^{p}(\Omega)$ for some $1 \leq p<\infty$, then there is a constant $C_{0}>0$ such that we both have

$$
\left\|\sum_{k=1}^{n} \varepsilon_{k} x_{k}\right\|_{\operatorname{Rad}\left(L^{p}(\Omega)\right)} \leq C_{0}\left\|\left(\sum_{k=1}^{n}\left|x_{k}\right|^{2}\right)^{1 / 2}\right\|_{L^{p}(\Omega)}
$$

and

$$
\left\|\left(\sum_{k=1}^{n}\left|x_{k}\right|^{2}\right)^{1 / 2}\right\|_{L^{p}(\Omega)} \leq C_{0}\left\|\sum_{k=1}^{n} \varepsilon_{k} x_{k}\right\|_{\operatorname{Rad}\left(L^{p}(\Omega)\right)}
$$

for any finite family $x_{1}, \ldots, x_{n}$ in $L^{p}(\Omega)$. Thus $\mathcal{T} \subset B\left(L^{p}(\Omega)\right)$ is $R$-bounded provided that

$$
\left\|\left(\sum_{k=1}^{n}\left|T_{k}\left(x_{k}\right)\right|^{2}\right)^{1 / 2}\right\|_{L^{p}(\Omega)} \leq C\left\|\left(\sum_{k=1}^{n}\left|x_{k}\right|^{2}\right)^{1 / 2}\right\|_{L^{p}(\Omega)}
$$

for some constant $C \geq 0$, and for any $T_{1}, \ldots, T_{n}$ in $\mathcal{T}$ and $x_{1}, \ldots, x_{n}$ in $L^{p}(\Omega)$. In the proof of Theorem 1.1, we shall need the following continuous version of (2.3) which was first observed by Weis [21, 4.a].

LEMMA 2.1. - Let $I \subset \mathbb{R}$ be an interval and let $S: I \rightarrow B\left(L^{p}(\Omega)\right)$ be a strongly continuous function, with $1 \leq p<\infty$. Then the set $\mathcal{T}=\{S(t) ; t \in I\}$ is $R$ bounded if and only if there is a constant $C \geq 0$ such that

$$
\left\|\left(\int_{I}|S(t) u(t)|^{2} \mathrm{~d} t\right)^{1 / 2}\right\|_{L^{p}(\Omega)} \leq C\left\|\left(\int_{I}|u(t)|^{2} \mathrm{~d} t\right)^{1 / 2}\right\|_{L^{p}(\Omega)}
$$

for any $u \in L^{p}\left(\Omega ; L^{2}(I)\right)$. Moreover the smallest possible $C$ is equivalent to $R(\mathcal{T})$.

We will also use the following well-known consequence of [4, Lemma 3.2].

Lemma 2.2. - Let $I \subset \mathbb{R}$ be an interval and let $\mathcal{T} \subset B\left(L^{p}(\Omega)\right)$ be an $R$ bounded set, with $1 \leq p<\infty$. Then the set

$$
\left\{\int_{I} a(r) R(r) \mathrm{d} r ; R: I \rightarrow \mathcal{T} \quad \text { is continuous, } a \in L^{1}(I) \text { and }\|a\|_{1} \leq 1\right\}
$$

is $R$-bounded as well and its $R$-boundedness constant is $\leq 2 R(\mathcal{T})$.

We finally recall some well-known facts concerning $H_{0}^{\infty}\left(\Sigma_{\theta}\right)$ that will be used without further reference. First of all, if $\varphi \in H_{0}^{\infty}\left(\Sigma_{\theta}\right)$ and $A$ is a sectorial operator of type $\omega<\theta$ on $X$, then $t \mapsto \varphi(t A)$ is a continuous and bounded function from $(0, \infty)$ into $B(X)$. Second, if $\gamma<\theta$ then $\int_{\Gamma_{\gamma}}|\varphi(z)| \cdot|\mathrm{d} z / z|<\infty$ by (1.1). Third, changing $z$ into $t z$ shows that

$$
\int_{\Gamma_{\gamma}}|\varphi(t z)| \cdot\left|\frac{\mathrm{d} z}{z}\right|=\int_{\Gamma_{\gamma}}|\varphi(z)| \cdot\left|\frac{\mathrm{d} z}{z}\right|
$$

TOME $132-2004-\mathrm{N}^{\mathrm{O}} 1$ 
for any $t>0$. Fourth, a simple change of variables also shows that

$$
\sup _{z \in \Gamma_{\gamma}} \int_{0}^{\infty}|\varphi(t z)| \frac{\mathrm{d} t}{t}<\infty .
$$

Proof of Theorem 1.1. - The proof is a generalization of the one of [19, Theorem 5]. By assumption, $A$ is an $R$-sectorial operator of $R$-type $\omega \in(0, \pi)$ on $L^{p}(\Omega)$ and we consider $F, G \in H_{0}^{\infty}\left(\Sigma_{\theta}\right) \backslash\{0\}$ for some $\theta \in(\omega, \pi)$. Note that the second assertion follows from the first one in Theorem 1.1. Indeed applying 1) with the constant function $f(z)=1$ yields an estimate $\|x\|_{F} \leq K\|x\|_{G}$. Then 2) follows by switching the roles of $F$ and $G$. Also observe that to prove 1), we may assume that $f \in H_{0}^{\infty}\left(\Sigma_{\theta}\right)$. Indeed assume (1.3) for any element of $H_{0}^{\infty}\left(\Sigma_{\theta}\right)$, and let $f \in H^{\infty}\left(\Sigma_{\theta}\right)$ be an arbitrary function. Then according to the so-called Convergence Lemma (see [6, Lemma 2.1]), there exists a constant $C>0$ not depending on $f$ and a bounded sequence $\left(f_{n}\right)_{n \geq 1} \subset H_{0}^{\infty}\left(\Sigma_{\theta}\right)$ such that $\left\|f_{n}\right\|_{\infty, \theta} \leq$ $C\|f\|_{\infty, \theta}$ for any $n \geq 1$ and $\lim _{n \rightarrow \infty}\left\|f_{n}(A) \bar{F}(t A) x-f(A) F(t A) x\right\|=0$ for any $x \in X$ and any $t>0$. Applying Fatou's Lemma, we may therefore deduce that

$$
\begin{gathered}
\left\|\left(\int_{0}^{\infty}|f(A) F(t A) x|^{2} \frac{\mathrm{d} t}{t}\right)^{1 / 2}\right\|_{p} \leq \liminf _{n \rightarrow \infty}\left\|\left(\int_{0}^{\infty}\left|f_{n}(A) F(t A) x\right|^{2} \frac{\mathrm{d} t}{t}\right)^{1 / 2}\right\|_{p} \\
\leq K \liminf _{n \rightarrow \infty}\left\|f_{n}\right\|_{\infty, \theta}\left\|\left(\int_{0}^{\infty}|G(t A) x|^{2} \frac{\mathrm{d} t}{t}\right)^{1 / 2}\right\|_{p} \\
\leq K C\|f\|_{\infty, \theta}\left\|\left(\int_{0}^{\infty}|G(t A) x|^{2} \frac{\mathrm{d} t}{t}\right)^{1 / 2}\right\|_{p}
\end{gathered}
$$

Throughout the rest of this proof, $x$ will be an element of $L^{p}(\Omega)$ such that $\|x\|_{G}<\infty$ and $f$ will be an element of $H_{0}^{\infty}\left(\Sigma_{\theta}\right)$. We will denote by $C_{1}, C_{2}, C_{3}, \ldots$ various constants not depending either on $f$ or on $x$. We fix an angle $\gamma \in(\omega, \theta)$ for which we will use the integral representation (1.2). We record for further use that by our $R$-sectoriality assumption, the set

$$
\left\{z R(z, A) ; z \in \Gamma_{\gamma}\right\} \quad \text { is } R \text {-bounded. }
$$

Then we consider two auxiliary functions $\varphi$ and $\psi$ in $H_{0}^{\infty}\left(\Sigma_{\theta}\right)$ such that

$$
\int_{0}^{\infty} \varphi(t) \psi(t) G(t) \frac{\mathrm{d} t}{t}=1
$$

We will reach (1.3) after five steps, the identity (2.5) being used only in the last one.

First step. - By (1.2) we have for any $t>0$

$$
f(A) \psi(t A)=\frac{1}{2 \pi i} \int_{\Gamma_{\gamma}} f(z) \psi(t z) z R(z, A) \frac{\mathrm{d} z}{z} .
$$

BULLETiN DE LA SOCiÉtÉ MATHÉMATiQUe DE FRANCE 
Moreover, letting $C_{1}=\int_{\Gamma_{\gamma}}|\psi(z)| \cdot|\mathrm{d} z / z|$, we have

$$
\int_{\Gamma_{\gamma}}|f(z) \psi(t z)| \cdot\left|\frac{\mathrm{d} z}{z}\right| \leq\|f\|_{\infty, \theta} \int_{\Gamma_{\gamma}}|\psi(t z)| \cdot\left|\frac{\mathrm{d} z}{z}\right|=C_{1}\|f\|_{\infty, \theta} .
$$

By Lemma 2.2 and (2.4), we therefore deduce that the operators $f(A) \psi(t A)$ form an $R$-bounded set and that we have an estimate

$$
R(\{f(A) \psi(t A) ; t>0\}) \leq C_{2}\|f\|_{\infty, \theta} .
$$

Hence applying Lemma 2.1 with $I=(0, \infty), S(t)=f(A) \psi(t A)$, and $u(t)=$ $G(t A) x / \sqrt{t}$, we obtain an estimate

$$
\left\|\left(\int_{0}^{\infty}|f(A) \psi(t A) G(t A) x|^{2} \frac{\mathrm{d} t}{t}\right)^{1 / 2}\right\|_{p} \leq C_{3}\|f\|_{\infty, \theta} \cdot\|x\|_{G} .
$$

Second step. - We define a continuous function $u: \Gamma_{\gamma} \rightarrow L^{p}(\Omega)$ by letting

$$
u(z)=\int_{0}^{\infty} \varphi(t z) f(A) \psi(t A) G(t A) x \frac{\mathrm{d} t}{t}, \quad z \in \Gamma_{\gamma} .
$$

Letting $w(t)=f(A) \psi(t A) G(t A) x$ for $t>0$, we see using the Cauchy-Schwarz inequality and Fubini's Theorem that $u$ satisfies the following pointwise estimates:

$$
\begin{aligned}
\int_{\Gamma_{\gamma}}|u(z)|^{2} \cdot\left|\frac{\mathrm{d} z}{z}\right| & \leq \int_{\Gamma_{\gamma}}\left(\int_{0}^{\infty}|\varphi(t z)| \cdot|w(t)| \frac{\mathrm{d} t}{t}\right)^{2}\left|\frac{\mathrm{d} z}{z}\right| \\
& \leq \int_{\Gamma_{\gamma}}\left(\int_{0}^{\infty}|\varphi(t z)| \frac{\mathrm{d} t}{t}\right)\left(\int_{0}^{\infty}|\varphi(t z)| \cdot|w(t)|^{2} \frac{\mathrm{d} t}{t}\right)\left|\frac{\mathrm{d} z}{z}\right| \\
& \leq\left(\sup _{z \in \Gamma_{\gamma}} \int_{0}^{\infty}|\varphi(t z)| \frac{\mathrm{d} t}{t}\right) \int_{0}^{\infty} \int_{\Gamma_{\gamma}}|\varphi(t z)| \cdot|w(t)|^{2} \cdot\left|\frac{\mathrm{d} z}{z}\right| \frac{\mathrm{d} t}{t} \\
& \leq\left(\sup _{z \in \Gamma_{\gamma}} \int_{0}^{\infty}|\varphi(t z)| \frac{\mathrm{d} t}{t}\right)\left(\sup _{t>0} \int_{\Gamma_{\gamma}}|\varphi(t z)| \cdot\left|\frac{\mathrm{d} z}{z}\right|\right) \int_{0}^{\infty}|w(t)|^{2} \frac{\mathrm{d} t}{t} .
\end{aligned}
$$

According to the discussion preceding this proof, the two suprema appearing here are finite hence applying (2.6) yields an estimate

$$
\left\|\left(\int_{\Gamma_{\gamma}}|u(z)|^{2} \cdot\left|\frac{\mathrm{d} z}{z}\right|\right)^{1 / 2}\right\|_{p} \leq C_{4}\|f\|_{\infty, \theta} \cdot\|x\|_{G} .
$$

Third step. - We now apply Lemma 2.1 with $I=\Gamma_{\gamma}$ and $S(z)=z R(z, A)$. By (2.4) and (2.8), we obtain a new estimate

$$
\left\|\left(\int_{\Gamma_{\gamma}}|z R(z, A) u(z)|^{2} \cdot\left|\frac{\mathrm{d} z}{z}\right|\right)^{1 / 2}\right\|_{p} \leq C_{5}\|f\|_{\infty, \theta} \cdot\|x\|_{G} .
$$

TOME $132-2004-\mathrm{N}^{\mathrm{O}} 1$ 
Fourth step. - This fourth step is similar to the second one. We define a continuous function $v:(0, \infty) \rightarrow L^{p}(\Omega)$ by letting

$$
v(s)=\frac{1}{2 \pi i} \int_{\Gamma_{\gamma}} F(s z) R(z, A) u(z) \mathrm{d} z, \quad s>0 .
$$

Then arguing as in the second step we find a constant $C_{6} \geq 0$ such that

$$
\left\|\left(\int_{0}^{\infty}|v(s)|^{2} \frac{\mathrm{d} s}{s}\right)^{1 / 2}\right\|_{p} \leq C_{6}\left\|\left(\int_{\Gamma_{\gamma}}|z R(z, A) u(z)|^{2} \cdot\left|\frac{\mathrm{d} z}{z}\right|\right)^{1 / 2}\right\|_{p} .
$$

Combining with (2.9), we obtain the final estimate

$$
\left\|\left(\int_{0}^{\infty}|v(s)|^{2} \frac{\mathrm{d} s}{s}\right)^{1 / 2}\right\|_{p} \leq C_{7}\|f\|_{\infty, \theta} \cdot\|x\|_{G} .
$$

Fifth step. - We conclude our proof by showing that for any $s>0$, $f(A) F(s A) x=v(s)$. By the Principle of Analytic Continuation, (2.5) implies that for any $z \in \Sigma_{\theta}$,

$$
\int_{0}^{\infty} \varphi(t z) \psi(t z) G(t z) \frac{\mathrm{d} t}{t}=1
$$

Since $f \in H_{0}^{\infty}\left(\Sigma_{\theta}\right)$, we deduce by applying (1.2) and Fubini's Theorem that

$$
f(A)=\int_{0}^{\infty} \varphi(t A) \psi(t A) G(t A) f(A) \frac{\mathrm{d} t}{t},
$$

the latter integral being absolutely convergent. Therefore we have for any $s>0$,

$$
\begin{aligned}
f(A) F(s A) x & =\int_{0}^{\infty} F(s A) \varphi(t A) \psi(t A) G(t A) f(A) x \frac{\mathrm{d} t}{t} \\
& =\int_{0}^{\infty}\left(\frac{1}{2 \pi i} \int_{\Gamma_{\gamma}} F(s z) \varphi(t z) R(z, A) \mathrm{d} z\right) \psi(t A) G(t A) f(A) x \frac{\mathrm{d} t}{t}
\end{aligned}
$$

by $(1.2)$,

$$
=\frac{1}{2 \pi i} \int_{\Gamma_{\gamma}} F(s z) R(z, A)\left(\int_{0}^{\infty} \varphi(t z) \psi(t A) G(t A) f(A) x \frac{\mathrm{d} t}{t}\right) \mathrm{d} z
$$

by Fubini's Theorem,

$$
\begin{aligned}
& =\frac{1}{2 \pi i} \int_{\Gamma_{\gamma}} F(s z) R(z, A) u(z) \mathrm{d} z \quad \text { by }(2.7) \text {, } \\
& =v(s) \quad \text { by }(2.10) \text {. }
\end{aligned}
$$

Assume now that $1<p<\infty$ and let $A$ be a sectorial operator on $L^{p}(\Omega)$ with a bounded $H^{\infty}\left(\Sigma_{\theta}\right)$ functional calculus. The following two results were proved by Cowling-Doust-McIntosh-Yagi [6, Section 6]. First, for any $F \in H_{0}^{\infty}\left(\Sigma_{\theta+}\right)$, there is a constant $K>0$ such that $\|x\|_{F} \leq K\|x\|$ for any $x \in L^{p}(\Omega)$. Second, there exists $F \in H_{0}^{\infty}\left(\Sigma_{\theta+}\right)$ as above such that for some suitable $K>0$, we have $K^{-1}\|x\| \leq\|x\|_{F} \leq K\|x\|$ for any $x \in L^{p}(\Omega)$. On the other hand, it follows from [14, Theorem 5.3] that $A$ is $R$-sectorial of $R$-type $\theta$ provided that $A$ has a 
bounded $H^{\infty}\left(\Sigma_{\theta}\right)$ functional calculus. Combining with Theorem 1.1, we deduce the following strengthening of the above mentioned result.

Corollary 2.3. - Let $A$ be a sectorial operator with a bounded $H^{\infty}\left(\Sigma_{\theta}\right)$ functional calculus on $L^{p}(\Omega)$, with $1<p<\infty$. Then for any $F \in H_{0}^{\infty}\left(\Sigma_{\theta+}\right)$, there is a constant $K>0$ such that for any $x \in L^{p}(\Omega)$,

$$
K^{-1}\|x\| \leq\left\|\left(\int_{0}^{\infty}|F(t A) x|^{2} \frac{\mathrm{d} t}{t}\right)^{1 / 2}\right\|_{p} \leq K\|x\| .
$$

REMARK 2.4. - The above corollary clearly has a converse. Indeed assume that $A$ is $R$-sectorial of $R$-type $\omega$ and satisfies the equivalence (2.11) for some $\theta>\omega$ and some $F \in H_{0}^{\infty}\left(\Sigma_{\theta}\right)$. Then applying the first part of Theorem 1.1 with $F=G$, we see that $A$ admits a bounded $H^{\infty}\left(\Sigma_{\theta}\right)$ functional calculus. This leads to the question of computing square functions for $R$-sectorial operators without a bounded $H^{\infty}$ functional calculus. We give a simple example below.

ExAmple 2.5. - Let $1<p \neq 2<\infty$ and let $\mathbb{T}=\{z \in \mathbb{C} ;|z|=1\}$ be the unimodular complex group equipped with its Haar measure. For any integer $n \in \mathbb{Z}$, we let $e_{n}(z)=z^{n}$ for $z \in \mathbb{T}$. As far as we know, the simplest example of a sectorial operator on an $L^{p}$-space without a bounded $H^{\infty}$ functional calculus is obtained by defining $A$ as the Fourier multiplier associated to the sequence $\left(2^{n}\right)_{n}$ on $L^{p}(\mathbb{T})$. Namely we let $A$ be the closure of the operator defined on $\operatorname{Span}\left\{e_{n} ; n \in \mathbb{Z}\right\}$ by first taking $e_{n}$ to $2^{n} e_{n}$ for any $n$ and then extending by linearity. This operator is essentialy the discrete version of the one given in $[6$, Example 5.2]. The arguments given in the latter paper extend to this discrete version and show that our operator $A$ is sectorial of any positive type, has no bounded $H^{\infty}$ functional calculus and admits bounded imaginary powers with $\left\|A^{i s}\right\|=1$ for any $s \in \mathbb{R}$. According to [5, Theorem 4] or [22], this implies that $A$ is $R$-sectorial of $R$-type $\omega$ for any $\omega>0$. Hence by Theorem 1.1, all non zero square function norms associated to $A$ are equivalent. We claim that they are actually all equivalent to the norm of $L^{2}(\mathbb{T})$. Here is a brief proof using Theorem 1.1. We give ourselves some $\theta>0$ and some $F \in H_{0}^{\infty}\left(\Sigma_{\theta}\right) \backslash\{0\}$. We let $\left(\alpha_{n}\right)_{n}$ be a finite sequence of complex numbers and consider $x=\sum_{n} \alpha_{n} e_{n}$. For any $z \in \mathbb{T}$ and any $t>0$, we have

$$
(F(t A) x)(z)=\sum_{n} F\left(t 2^{n}\right) \alpha_{n} e_{n}(z)
$$

Likewise for every $f \in H^{\infty}\left(\Sigma_{\theta}\right)$, we have

$$
(f(A) F(t A) x)(z)=\sum_{n} f\left(2^{n}\right) F\left(t 2^{n}\right) \alpha_{n} e_{n}(z) .
$$

TOME $132-2004-\mathrm{N}^{\mathrm{O}} 1$ 
Hence if we let $\Lambda=L^{p}\left(\mathbb{T} ; L^{2}(0, \infty ; \mathrm{d} t / t)\right)$ and apply (1.3) with $F=G$, we obtain that

$$
\left\|\sum_{n} f\left(2^{n}\right) F\left(t 2^{n}\right) \alpha_{n} e_{n}(z)\right\|_{\Lambda} \leq K\|f\|_{\infty, \theta} \cdot\left\|\sum_{n} F\left(t 2^{n}\right) \alpha_{n} e_{n}(z)\right\|_{\Lambda} .
$$

Now using the fact that $\left(2^{n}\right)_{n}$ is an interpolation sequence for the open set $\Sigma_{\theta}$, we deduce that for an appropriate constant $K_{1}>0$, we have

$$
\left\|\sum_{n} \varepsilon_{n} F\left(t 2^{n}\right) \alpha_{n} e_{n}(z)\right\|_{\Lambda} \leq K_{1}\left\|\sum_{n} F\left(t 2^{n}\right) \alpha_{n} e_{n}(z)\right\|_{\Lambda}
$$

for any $\{-1,1\}$-valued sequence $\left(\varepsilon_{n}\right)_{n}$ (see e.g. [7, Chapter VII] for details). Taking the average over all such possible sequences we find that

$$
\left\|\sum_{n} F\left(t 2^{n}\right) \alpha_{n} e_{n}(z)\right\|_{\Lambda} \asymp\left\|\sum_{n} \varepsilon_{n} F\left(t 2^{n}\right) \alpha_{n} e_{n}(z)\right\|_{\operatorname{Rad}(\Lambda)} .
$$

Using the well-known fact that (2.1) and (2.2) hold with $\Lambda$ in place of $L^{p}(\Omega)$ we finally obtain that

$$
\left\|\left(\int_{0}^{\infty}\left|F(t A)\left(\sum_{n} \alpha_{n} e_{n}\right)\right|^{2} \frac{\mathrm{d} t}{t}\right)^{1 / 2}\right\|_{p} \asymp\left\|\left(\sum_{n}\left|F\left(t 2^{n}\right) \alpha_{n} e_{n}(z)\right|^{2}\right)^{1 / 2}\right\|_{\Lambda} .
$$

Now observe that since $\left|e_{n}(z)\right|=1$ for any $z \in \mathbb{T}$ and $\int_{0}^{\infty}\left|F\left(t 2^{n}\right)\right|^{2} \mathrm{~d} t / t=$ $\int_{0}^{\infty}|F(t)|^{2} \mathrm{~d} t / t$ for any $n \in \mathbb{Z}$, we have

$$
\begin{aligned}
\left\|\left(\sum_{n}\left|F\left(t 2^{n}\right) \alpha_{n} e_{n}(z)\right|^{2}\right)^{1 / 2}\right\|_{\Lambda}^{2} & =\left\|\left(\sum_{n}\left|F\left(t 2^{n}\right) \alpha_{n}\right|^{2}\right)^{1 / 2}\right\|_{L^{2}(0, \infty ; \mathrm{d} t / t)}^{2} \\
& =\left(\int_{0}^{\infty}|F(t)|^{2} \frac{\mathrm{d} t}{t}\right) \sum_{n}\left|\alpha_{n}\right|^{2} \\
& =\left(\int_{0}^{\infty}|F(t)|^{2} \frac{\mathrm{d} t}{t}\right)\left\|\sum_{n} \alpha_{n} e_{n}\right\|_{2}^{2}
\end{aligned}
$$

which proves the announced result.

REMARK 2.6. - It was observed in [15] that most of the results established in [6] extend to the case when $L^{p}(\Omega)$ is replaced by a $B$-convex Banach lattice. It is also easy to check that our Theorem 1.1 extends to this setting and as a by-product, we find that Corollary 2.3 also extends to this setting.

\section{Application to $R$-admissibility.}

Let $X$ be a Banach space and let $\left(T_{t}\right)_{t \geq 0}$ be a bounded $c_{0}$-semigroup on $X$. We let $-A$ denote its infinitesimal generator and we let $D(A)$ be the domain of $A$. We consider a linear mapping $C: D(A) \rightarrow Y$ valued in another Banach space $Y$. We assume that $C$ is continuous with respect to the graph norm of $D(A)$, so what $t \mapsto C T_{t}(x)$ is a well-defined continuous function from $(0, \infty)$ 
into $Y$ for any $x \in D(A)$. By definition, $C$ is admissible for $A$ if there is a constant $M>0$ such that

$$
\int_{0}^{\infty}\left\|C T_{t}(x)\right\|^{2} \mathrm{~d} t \leq M^{2}\|x\|^{2}, \quad x \in D(A) .
$$

This definition arises from Control Theory and is usually given with $X$ and $Y$ being Hilbert spaces. We refer the reader to [24], [23], [25], [8], [20], [9] and the references therein for some background and applications of this notion.

If $C$ is admissible for $A$, then there is a constant $K>0$ such that

$$
\left\|(-\operatorname{Re}(\lambda))^{1 / 2} C R(\lambda, A)\right\| \leq K, \quad \lambda \in \mathbb{C}, \operatorname{Re}(\lambda)<0 .
$$

Indeed if $\operatorname{Re}(\lambda)<0$, define

$$
a_{\lambda}(t)=-(-\operatorname{Re}(\lambda))^{1 / 2} \mathrm{e}^{\lambda t}, \quad t>0 .
$$

Then

$$
a_{\lambda} \in L^{2}(0, \infty ; d t) \quad \text { with } \quad\left\|a_{\lambda}\right\|_{2}=\frac{1}{\sqrt{2}},
$$

and according to the Laplace Formula, we have

$$
(-\operatorname{Re}(\lambda))^{1 / 2} C R(\lambda, A) x=\int_{0}^{\infty} a_{\lambda}(t) C T_{t}(x) \mathrm{d} t, \quad \operatorname{Re}(\lambda)<0,
$$

for any $x \in D(A)$. Thus (3.1) implies (3.2) with $K=M / \sqrt{2}$ by the CauchySchwarz inequality.

The latter observation goes back to George Weiss [25] who investigated the converse implication, that is, whether the estimate (3.2) implies that $C$ is admissible for $A$. He quickly proved that this converse does not hold on general Banach spaces but the question remained open for a long time under the name of "Weiss conjecture" in the case when $X$ and $Y$ are both Hilbert spaces. The Weiss conjecture has been disproved recently by Jacob-Partington-Pott [10]. Namely there exist Hilbert spaces $X, Y$, as well as $T_{t}=\mathrm{e}^{-t A}$ and $C$ as above such that (3.2) holds for some $K$ although $C$ is not admissible for $A$. In fact it was proved by Jacob-Zwart [12] that such counterexamples exist with $Y=\mathbb{C}$. See also [11] for related work. The failure of the Weiss conjecture leads to the following question.

Which triples $(X, A, Y)$ have the property that any continuous $C: D(A) \rightarrow Y$ is admissible for $A$ provided that (3.2) holds?

In [9], it was shown that this property holds when $X$ is a Hilbert space, $Y=\mathbb{C}$, and $A$ is maximal accretive (equivalently, $\left(T_{t}\right)_{t>0}$ is a contraction semigroup). In [16], we studied the case when $T_{t}=\mathrm{e}^{-t A}$ is a bounded analytic semigroup, that is, there exists $\alpha>0$ such that $\left(T_{t}\right)_{t>0}$ extends to a bounded analytic family $\left(\mathrm{e}^{-z A}\right)_{z \in \Sigma_{\alpha}} \subset B(X)$. We proved the following result (see [16, Theorem 4.1]). 
ThEOREM 3.1. - Assume that $T_{t}=\mathrm{e}^{-t A}$ is a bounded analytic semigroup on a Banach space $X$. Then the following assertions are equivalent.

(i) $A^{1 / 2}$ is admissible for $A$.

(ii) For any Banach space $Y$, a continuous mapping $C: D(A) \rightarrow Y$ is admissible for $A$ if and only if there is a constant $K>0$ such that $\left\|(-\operatorname{Re}(\lambda))^{1 / 2} C R(\lambda, A)\right\| \leq K$ for any $\lambda \in \mathbb{C}$ with $\operatorname{Re}(\lambda)<0$.

(iii) For any Banach space $Y$, a continuous mapping $C: D(A) \rightarrow Y$ is admissible for $A$ if and only if there is a constant $K>0$ such that $\left\|(-s)^{1 / 2} C R(s, A)\right\| \leq K$ for any negative real number $s<0$.

Recall that $T_{t}=\mathrm{e}^{-t A}$ is a bounded analytic semigroup on $X$ if and only if $A$ satisfies the conditions $(\mathrm{S} 1)$ and $(\mathrm{S} 2)$ from Section 1 for some $\omega<\frac{1}{2} \pi$. Define

$$
F_{0}(z)=z^{1 / 2} \mathrm{e}^{-z}, \quad z \in \mathbb{C} .
$$

Then $F_{0} \in H_{0}^{\infty}\left(\Sigma_{\theta}\right)$ for any $\theta \in\left(0, \frac{1}{2} \pi\right)$ and

$$
A^{1 / 2} T_{t}(x)=\frac{F_{0}(t A) x}{\sqrt{t}}, \quad t>0, x \in X .
$$

Consequently, $A^{1 / 2}$ is admissible for $A$ if and only if we have an estimate

$$
\left(\int_{0}^{\infty}\left\|F_{0}(t A) x\right\|^{2} \frac{\mathrm{d} t}{t}\right)^{1 / 2} \leq M\|x\|, \quad x \in X
$$

This observation makes Theorem 3.1 especially interesting in the case when $X=H$ is a Hilbert space. Indeed in that case, an appeal to [18] shows that condition (i), hence conditions (ii) and (iii) in Theorem 3.1 are fulfilled provided that $A$ admits a bounded $H^{\infty}$ functional calculus. We refer the reader to $[16$, Section 5] for a more precise discussion of condition (i) of Theorem 3.1 in the case when $X=H$ is a Hilbert space.

When moving from Hilbert spaces to $L^{p}$-spaces, it is natural to introduce a variant of admissibility involving square function norms in the style of those considered so far in the previous two sections. We let $1<p, q<\infty$ be two numbers, we let $\Omega$ and $\Sigma$ be two measure spaces and we let $\left(T_{t}\right)_{t>0}$ be a bounded $c_{0}$-semigroup on $L^{p}(\Omega)$ with generator denoted by $-A$. Then given a continuous linear mapping $C: D(A) \rightarrow L^{q}(\Sigma)$, we say that $C$ is $R$-admissible for $A$ if there is a constant $M>0$ such that

$$
\left\|\left(\int_{0}^{\infty}\left|C T_{t}(x)\right|^{2} d t\right)^{1 / 2}\right\|_{L^{q}(\Sigma)} \leq M\|x\|_{L^{p}(\Omega)}, \quad x \in D(A) .
$$

Arguing as above, it is easy to check that this condition implies (3.2) with $K=M / \sqrt{2}$. It turns out that the following stronger property holds.

Lemma 3.2. - If $C$ is $R$-admissible for $A$, then the following set is $R$-bounded:

$$
\left\{(-\operatorname{Re}(\lambda))^{1 / 2} C R(\lambda, A) ; \lambda \in \mathbb{C}, \operatorname{Re}(\lambda)<0\right\} .
$$


Indeed this follows from (3.3), (3.4) and the following statement of independent interest. Note the analogy with Lemma 2.2 .

Proposition 3.3. - Let $X$ be a Banach space, let $X_{0} \subset X$ be a dense subspace, and let $t \mapsto \varphi_{t}$ be a strongly continuous function from an interval $I \subset \mathbb{R}$ into the space $L\left(X_{0}, L^{q}(\Sigma)\right)$ of linear mappings from $X_{0}$ into $L^{q}(\Sigma)$. Assume that there is a constant $M>0$ such that

$$
\left\|\left(\int_{I}\left|\varphi_{t}(x)\right|^{2} \mathrm{~d} t\right)^{1 / 2}\right\|_{L^{q}(\Sigma)} \leq M\|x\|, \quad x \in X_{0}
$$

For any $a \in L^{2}(I)$, let $\int_{I} a(t) \varphi_{t} \mathrm{~d} t$ denote the element of $B\left(X, L^{q}(\Sigma)\right)$ obtained by first taking $x \in X_{0}$ to $\int_{I} a(t) \varphi_{t}(x) \mathrm{d} t \in L^{q}(\Sigma)$ and then extending by continuity. Then the following set is $R$-bounded:

$$
\left\{\int_{I} a(t) \varphi_{t} \mathrm{~d} t ; a \in L^{2}(I),\|a\|_{2} \leq 1\right\} .
$$

Proof. - We use the notation and definitions from the beginning of Section 2. For any $a \in L^{2}(I)$, we let

$$
T_{a}=\int_{I} a(t) \varphi_{t} \mathrm{~d} t
$$

and we give ourselves a finite family $a_{1}, \ldots, a_{n}$ of elements of $L^{2}(I)$ of norms less than or equal to one. Let $\left(e_{1}, \ldots, e_{m}\right)$ be an orthonormal basis of $\operatorname{Span}\left\{a_{1}, \ldots, a_{n}\right\} \subset L^{2}(I)$. Then we have $a_{k}=\sum_{i}\left\langle a_{k}, e_{i}\right\rangle e_{i}$ for any $k$, hence

$$
T_{a_{k}}=\sum_{i=1}^{m}\left\langle a_{k}, e_{i}\right\rangle T_{e_{i}}, \quad 1 \leq k \leq n .
$$

Let $x_{1}, \ldots, x_{n}$ be arbitrary elements of $X_{0}$. (Strictly speaking, we should take elements of $X$ but the density of $X_{0}$ clearly allows this reduction.) Then for some numerical constant $C_{0} \geq 0$, we have

$$
\begin{aligned}
\left\|\sum_{k=1}^{n} \varepsilon_{k} T_{a_{k}}\left(x_{k}\right)\right\|_{\operatorname{Rad}\left(L^{q}\right)} & \leq C_{0}\left\|\left(\sum_{k=1}^{n}\left|T_{a_{k}}\left(x_{k}\right)\right|^{2}\right)^{1 / 2}\right\|_{L^{q}} \\
& =C_{0}\left\|\left(\sum_{k=1}^{n}\left|\sum_{i=1}^{m}\left\langle a_{k}, e_{i}\right\rangle T_{e_{i}}\left(x_{k}\right)\right|^{2}\right)^{1 / 2}\right\|_{L^{q}}
\end{aligned}
$$

Using the Cauchy-Schwarz inequality, we obtain the following pointwise estimates on $L^{q}(\Sigma)$.

$$
\begin{aligned}
\sum_{k=1}^{n}\left|\sum_{i=1}^{m}\left\langle a_{k}, e_{i}\right\rangle T_{e_{i}}\left(x_{k}\right)\right|^{2} & \leq \sum_{k=1}^{n}\left(\sum_{i=1}^{m}\left|\left\langle a_{k}, e_{i}\right\rangle\right|^{2}\right)\left(\sum_{i=1}^{m}\left|T_{e_{i}}\left(x_{k}\right)\right|^{2}\right) \\
& =\sum_{k=1}^{n}\left\|a_{k}\right\|_{2}^{2}\left(\sum_{i=1}^{m}\left|T_{e_{i}}\left(x_{k}\right)\right|^{2}\right) \leq \sum_{i, k}\left|T_{e_{i}}\left(x_{k}\right)\right|^{2}
\end{aligned}
$$

TOME $132-2004-\mathrm{N}^{\mathrm{O}} 1$ 
Combining with the preceding estimate, this yields

$$
\left\|\sum_{k=1}^{n} \varepsilon_{k} T_{a_{k}}\left(x_{k}\right)\right\|_{\operatorname{Rad}\left(L^{q}\right)} \leq C_{0}\left\|\left(\sum_{i, k}\left|T_{e_{i}}\left(x_{k}\right)\right|^{2}\right)^{1 / 2}\right\|_{L^{q}} .
$$

Now observe that since $\left(e_{1}, \ldots, e_{m}\right)$ is an orthonormal family of $L^{2}(I)$, we have

$$
\sum_{i}\left|\int_{I} e_{i}(t) \alpha(t) \mathrm{d} t\right|^{2} \leq \int_{I}|\alpha(t)|^{2} \mathrm{~d} t
$$

for any $\alpha \in L^{2}(I)$, hence we have a pointwise inequality

$$
\sum_{i}\left|T_{e_{i}}(x)\right|^{2} \leq \int_{I}\left|\varphi_{t}(x)\right|^{2} \mathrm{~d} t
$$

for any $x \in X_{0}$. Applying this to each $x_{k}$, we deduce that

$$
\sum_{i, k}\left|T_{e_{i}}\left(x_{k}\right)\right|^{2} \leq \int_{I} \sum_{k}\left|\varphi_{t}\left(x_{k}\right)\right|^{2} \mathrm{~d} t
$$

Since $\left(\varepsilon_{1}, \ldots, \varepsilon_{n}\right)$ is an orthonormal family of $L^{2}\left(\Omega_{0}\right)$, the right handside of the latter inequality can be written as

$$
\int_{I} \sum_{k}\left|\varphi_{t}\left(x_{k}\right)\right|^{2} \mathrm{~d} t=\int_{I} \int_{\Omega_{0}}\left|\sum_{k} \varepsilon_{k}(s) \varphi_{t}\left(x_{k}\right)\right|^{2} \mathrm{~d} \mathbb{P}(s) \mathrm{d} t .
$$

Owing to the Khintchine-Kahane inequality (see e.g. [17, p. 74]), there is a numerical constant $C_{1} \geq 0$ such that

$$
\begin{aligned}
\left(\int_{\Omega_{0}} \int_{I}\left|\sum_{k} \varepsilon_{k}(s) \varphi_{t}\left(x_{k}\right)\right|^{2} \mathrm{~d}\right. & t \mathrm{~d} \mathbb{P}(s))^{1 / 2} \\
& \leq C_{1} \int_{\Omega_{0}}\left(\int_{I}\left|\sum_{k} \varepsilon_{k}(s) \varphi_{t}\left(x_{k}\right)\right|^{2} \mathrm{~d} t\right)^{1 / 2} \mathrm{~d} \mathbb{P}(s) .
\end{aligned}
$$

We therefore obtain that

$$
\begin{aligned}
\left(\sum_{i, k}\left|T_{e_{i}}\left(x_{k}\right)\right|^{2}\right)^{1 / 2} & \leq C_{1} \int_{\Omega_{0}}\left(\int_{I}\left|\sum_{k} \varepsilon_{k}(s) \varphi_{t}\left(x_{k}\right)\right|^{2} \mathrm{~d} t\right)^{1 / 2} \mathrm{~d} \mathbb{P}(s) \\
& =C_{1} \int_{\Omega_{0}}\left(\int_{I}\left|\varphi_{t}\left(\sum_{k} \varepsilon_{k}(s) x_{k}\right)\right|^{2} \mathrm{~d} t\right)^{1 / 2} \mathrm{~d} \mathbb{P}(s) .
\end{aligned}
$$

Hence by (3.6), we deduce that

$$
\begin{aligned}
\left\|\sum_{k=1}^{n} \varepsilon_{k} T_{a_{k}}\left(x_{k}\right)\right\|_{\operatorname{Rad}\left(L^{q}\right)} & \leq C_{0} C_{1}\left\|\int_{\Omega_{0}}\left(\int_{I}\left|\varphi_{t}\left(\sum_{k} \varepsilon_{k}(s) x_{k}\right)\right|^{2} \mathrm{~d} t\right)^{1 / 2} \mathrm{~d} \mathbb{P}(s)\right\|_{L^{q}} \\
& \leq C_{0} C_{1} \int_{\Omega_{0}}\left\|\left(\int_{I}\left|\varphi_{t}\left(\sum_{k} \varepsilon_{k}(s) x_{k}\right)\right|^{2} \mathrm{~d} t\right)^{1 / 2}\right\|_{L^{q}} \mathrm{~d} \mathbb{P}(s) .
\end{aligned}
$$

BULletin DE LA SOCiÉtÉ MATHÉMATIQUE DE FRANCE 
It now remains to apply our assumption with $x=\sum_{k} \varepsilon_{k}(s) x_{k}$ for each $s \in \Omega_{0}$ to deduce that

$$
\left\|\sum_{k=1}^{n} \varepsilon_{k} T_{a_{k}}\left(x_{k}\right)\right\|_{\operatorname{Rad}\left(L^{q}\right)} \leq C_{0} C_{1} M\left\|\sum_{k} \varepsilon_{k} x_{k}\right\|_{\operatorname{Rad}(X)}
$$

which proves our $R$-boundedness property.

We record here the simple consequence of Lemma 3.2.

LEMmA 3.4. - If $C$ is $R$-admissible for $A$, then the set

$$
\left\{(-s)^{1 / 2} C R(s, A) ; s \in \mathbb{R}, s<0\right\}
$$

is R-bounded. The latter condition is equivalent to the existence of a constant $K>0$ such that

$$
\left\|\left(\int_{0}^{\infty}\left|C(t+A)^{-1} u(t)\right|^{2} \mathrm{~d} t\right)^{1 / 2}\right\|_{L^{q}(\Sigma)} \leq K\left\|\left(\int_{0}^{\infty}|u(t)|^{2} \frac{\mathrm{d} t}{t}\right)^{1 / 2}\right\|_{L^{p}(\Omega)}
$$

for any $u \in L^{p}\left(\Omega ; L^{2}(0, \infty ; \mathrm{d} t / t)\right)$.

Proof. - The first part follows from Lemma 3.2 whereas the second part follows by simply adapting the proof of Lemma 2.1 to the case of a function valued in $B\left(L^{p}(\Omega), L^{q}(\Sigma)\right)$. We skip the details.

We now come to the main result of this section, which is an analogue of Theorem 3.1 for $R$-admissibility. We will say that a bounded analytic semigroup $T_{t}=\mathrm{e}^{-t A}$ on $X$ is an $R$-bounded one if there exists $\alpha>0$ such that the set $\left\{\mathrm{e}^{-z A} ; z \in \Sigma_{\alpha}\right\} \subset B(X)$ is $R$-bounded. According to [22], this is equivalent to the existence of $\theta<\frac{1}{2} \pi$ such that $\left\{z R(z, A) ; z \in \bar{\Sigma}_{\theta}^{c}\right\}$ is $R$-bounded, hence (modulo (S3)) to the property that $A$ is $R$-sectorial of $R$-type $<\frac{1}{2} \pi$.

Note that according to the comments following Theorem 3.1, if $T_{t}=\mathrm{e}^{-t A}$ is a bounded analytic semigroup on $L^{p}(\Omega)$, then $A^{1 / 2}$ is $R$-admissible for $A$ if and only if there is a constant $M>0$ such that

$$
\|x\|_{F_{0}}=\left\|\left(\int_{0}^{\infty}\left|F_{0}(t A) x\right|^{2} \frac{\mathrm{d} t}{t}\right)^{1 / 2}\right\|_{L^{p}(\Omega)} \leq M\|x\|_{L^{p}(\Omega)}, \quad x \in L^{p}(\Omega) .
$$

Here $F_{0}$ is defined by $F_{0}(z)=z^{1 / 2} \mathrm{e}^{-z}$.

TheOREM 3.5. - Let $T_{t}=\mathrm{e}^{-t A}$ be an R-bounded analytic semigroup on $L^{p}(\Omega)$, with $1<p<\infty$. Then the following assertions are equivalent.

(i) $A^{1 / 2}$ is $R$-admissible for $A$.

(ii) For any $1<q<\infty$ and any measure space $\Sigma$, a continuous mapping $C: D(A) \rightarrow L^{q}(\Sigma)$ is R-admissible for $A$ if and only if the set $\left\{(-\operatorname{Re}(\lambda))^{1 / 2} C R(\lambda, A) ; \lambda \in \mathbb{C}, \operatorname{Re}(\lambda)<0\right\}$ is $R$-bounded.

TOME $132-2004-\mathrm{N}^{\mathrm{O}} 1$ 
(iii) For any $1<q<\infty$ and any measure space $\Sigma$, a continuous mapping $C: D(A) \rightarrow L^{q}(\Sigma)$ is $R$-admissible for $A$ if and only if the set $\left\{(-s)^{1 / 2} C R(s, A) ; s \in \mathbb{R}, s<0\right\}$ is $R$-bounded if and only if there is a constant $K>0$ such that

$$
\left\|\left(\int_{0}^{\infty}\left|C(t+A)^{-1} u(t)\right|^{2} \mathrm{~d} t\right)^{1 / 2}\right\|_{L^{q}(\Sigma)} \leq K\left\|\left(\int_{0}^{\infty}|u(t)|^{2} \frac{\mathrm{d} t}{t}\right)^{1 / 2}\right\|_{L^{p}(\Omega)}
$$

for any $u \in L^{p}\left(\Omega ; L^{2}(0, \infty ; \mathrm{d} t / t)\right)$.

Proof. - Owing to the results proved before, the proof is now a simple adaptation of that of Theorem 3.1 (stated as Theorem 4.1 in [16]). We shall therefore only sketch it. It is well-known that since $X=L^{p}(\Omega)$ is reflexive, it is the direct sum of the kernel of $A$ and of the closure of the range of $A$ hence we may clearly assume that $A$ has a dense range. We let $\omega<\frac{1}{2} \pi$ be such that $A$ is $R$-sectorial of $R$-type $\omega$.

It is obvious that (iii) implies (ii). To prove that (ii) implies (i), it suffices to show that the set

$$
\left\{|\lambda|^{1 / 2} A^{1 / 2} R(\lambda, A) ; \lambda \in \mathbb{C}, \operatorname{Re}(\lambda)<0\right\}
$$

is $R$-bounded. For we fix some angle $\gamma \in\left(\omega, \frac{1}{2} \pi\right)$ and we write

$$
(-\lambda)^{1 / 2} A^{1 / 2} R(\lambda, A)=\frac{1}{2 \pi i} \int_{\Gamma_{\gamma}} \frac{(-\lambda)^{1 / 2} z^{1 / 2}}{\lambda-z} R(z, A) \mathrm{d} z, \quad \lambda \in \mathbb{C}, \operatorname{Re}(\lambda)<0 .
$$

Since the set $\left\{z R(z, A) ; z \in \Gamma_{\gamma}\right\}$ is $R$-bounded, Lemma 2.2 ensures that it suffices to prove that for a certain constant $K>0$, we have

$$
\int_{\Gamma_{\gamma}} \frac{|\lambda z|^{1 / 2}}{|\lambda-z|} \cdot\left|\frac{\mathrm{d} z}{z}\right| \leq K, \quad \lambda \in \mathbb{C}, \operatorname{Re}(\lambda)<0 .
$$

This estimate holds true and is established in the course of the proof of $[16$, Theorem 4.1].

We now assume that $A^{1 / 2}$ is $R$-admissible for $A$ and will prove (iii). We consider a continuous mapping $C: D(A) \rightarrow L^{q}(\Sigma)$. In view of Lemma 3.4, we only need to prove that if the set

$$
\left\{(-s)^{1 / 2} C R(s, A) ; s \in \mathbb{R}, s<0\right\}
$$

is $R$-bounded, then $C$ is $R$-admissible for $A$. Arguing as in the proof of [16, Lemma 2.3], we obtain that the $R$-boundedness of (3.8) implies the existence of an angle $\nu \in(\omega, \pi)$ such that

$$
\left\{|z|^{1 / 2} C R(z, A) ;|\operatorname{Arg}(z)| \geq \nu\right\}
$$

is $R$-bounded as well. Then arguing as in the proof of [16, Theorem 4.1], we find $\theta \in(\nu, \pi)$ and functions $F_{1}, F_{2} \in H_{0}^{\infty}\left(\Sigma_{\omega+}\right)$ and $G_{1}, G_{2} \in H_{0}^{\infty}\left(\Sigma_{\theta}\right)$ such 
that $F_{0}=G_{1} F_{1}+G_{2} F_{2}$. According to (3.5), this yields

$$
C T_{t}(x)=\left[C A^{-1 / 2} G_{1}(t A)\right] \frac{F_{1}(t A) x}{\sqrt{t}}+\left[C A^{-1 / 2} G_{2}(t A)\right] \frac{F_{2}(t A) x}{\sqrt{t}}
$$

for any $t>0$ and every $x \in D(A)$. By our assumption (i), the estimate (3.7) holds for some $M>0$. We therefore deduce from Theorem 1.1 that for some constants $M_{1}, M_{2}>0$, we also have estimates

$$
\|x\|_{F_{1}} \leq M_{1}\|x\| \quad \text { and } \quad\|x\|_{F_{2}} \leq M_{2}\|x\| .
$$

As we already said, Lemma 2.1 extends to the case of functions valued in $B\left(L^{p}(\Omega), L^{q}(\Sigma)\right)$. Hence to deduce the $R$-admissibility of $C$ from (3.10) and (3.11), it now suffices to check that for $j=1,2$, the set

$$
\left\{C A^{-1 / 2} G_{j}(t A) ; t>0\right\}
$$

is $R$-bounded. According to the proof of [16, Theorem 4.1], each of the operators of the latter set has the following integral representation:

$$
C A^{-1 / 2} G_{j}(t A)=\frac{1}{2 \pi i} \int_{\Gamma_{\nu}} z^{-1 / 2} G_{j}(t z) C R(z, A) \mathrm{d} z .
$$

Since the set (3.9) is $R$-bounded and $\int_{\Gamma_{\nu}}\left|G_{j}(t z)\right| \cdot|\mathrm{d} z / z|=\int_{\Gamma_{\nu}}\left|G_{j}(z)\right| \cdot|\mathrm{d} z / z|<\infty$ for any $t>0$, we deduce from Lemma 2.2 that the set (3.12) is indeed $R$ bounded, which concludes our proof.

REMARK 3.6. - If $A$ is a sectorial operator on $L^{p}(\Omega)$ with a bounded $H^{\infty}\left(\Sigma_{\theta}\right)$ functional calculus for some $\theta<\frac{1}{2} \pi$, then it satisfies (3.7) by [6] hence $A^{1 / 2}$ is admissible for $A$. Furthermore it is $R$-sectorial of $R$-type $<\frac{1}{2} \pi$ by [14, Theorem 5.3], hence $T_{t}=\mathrm{e}^{-t A}$ is an $R$-bounded analytic semigroup. Consequently, $A$ satisfies the assertion (iii) of Theorem 3.5.

REMARK 3.7. - In [16, Section 5], we exhibited a sectorial operator $A_{0}$ on $\ell^{2}$ such that $A_{0}^{1 / 2}$ is admissible for $A_{0}$ although $A_{0}$ has no bounded $H^{\infty}$ functional calculus. Using the fact that $L^{p}(\mathbb{R})$, say, contains a complemented subspace isomorphic to $\ell^{2}$ when $1<p<\infty$, it is easy to transfer $A_{0}$ to an operator $A$ on $L^{p}(\mathbb{R})$ satisfying the assertions of Theorem 3.5 but having no bounded $H^{\infty}$ functional calculus.

\section{BIBLIOGRAPHY}

[1] Arendt (W.) \& Bu (S.) - The operator valued Marcinkiewicz multiplier theorem and maximal regularity, Math. Z., t. 240 (2002), pp. 311-343.

[2] Auscher (P.), Duong (X.T.) \& McIntosh (A.) - in preparation.

[3] Auscher (P.), McIntosh (A.) \& Nahmod (A.) - Holomorphic functional calculi of operators, quadratic estimates and interpolation, Indiana Univ. Math. J., t. 46 (1997), pp. 375-403.

TOME $132-2004-\mathrm{N}^{\mathrm{O}} 1$ 
[4] Clément (P.), De Pagter (B.), Sukochev (F.) \& Witvliet (H.) Schauder decompositions and multiplier theorems, Studia Math., t. 138 (2000), pp. 135-163.

[5] Clément (P.) \& Pruss (J.) - An operator valued transference principle and maximal regularity on vector valued $L_{p}$-spaces, in Proc. of the Sixth International Conference on Evolution Equations and their Applications in Physical and Life Sciences (Bad Herrenalb, 1998) (Lumer (G.) \& Weis (L.), eds.), Marcel Dekker, New-York, 2001, pp. 67-87.

[6] Cowling (M.), Doust (I.), McIntosh (A.) \& Yagi (A.) - Banach space operators with a bounded $H^{\infty}$ functional calculus, J. Austr. Math. Soc., t. 60 (1996), pp. 51-89.

[7] Garnett (J.B.) - Bounded analytic functions, Pure and applied Mathematics, vol. 96, Academic Press, 1981.

[8] Grabowsky (P.) \& Callier (F.M.) - Admissible observation operators. Semigroup criteria of admissibility, Int. Equ. Oper. Theory, t. 25 (1996), pp. $182-198$.

[9] Jacob (B.) \& Partington (J.R.) - The Weiss conjecture on admissibility of observation operators for contraction semigroups, Int. Equ. Oper. Theory, t. 40 (2001), pp. 231-243.

[10] Jacob (B.), Partington (J.R.) \& Potт (S.) - Admissible and weakly admissible observation operators for the right shift semigroup, Proc. Edinburgh Math. Soc., t. 45 (2002), pp. 353-362.

[11] Jacob (B.), Staffans (O.) \& Zwart (H.) - Weak admissibility does not imply admissibility for analytic semigroups, 2003.

[12] JaCOB (B.) \& Zwart (H.) - Disproof of two conjectures of George Weiss, Preprint, 2000.

[13] Kalton (N.) \& Lancien (G.) - A solution to the problem of $L^{p}$-maximal regularity, Math. Z., t. 235 (2000), pp. 559-568.

[14] Kalton (N.) \& Weis (L.) - The $H^{\infty}$ calculus and sums of closed operators, Math. Ann., t. 321 (2001), pp. 319-345.

[15] Lancien (F.), Lancien (G.) \& Le Merdy (C.) - A joint functional calculus for sectorial operators with commuting resolvents, Proc. London Math. Soc., t. 77 (1998), pp. 387-414.

[16] Le Merdy (C.) - The Weiss conjecture for bounded analytic semigroups, J. London Math. Soc. (2), t. 67 (2003), pp. 715-738.

[17] Lindenstrauss (J.) \& Tzafriri (L.) - Classical Banach spaces II, Springer Verlag, Berlin, 1979.

[18] McIntosh (A.) - Operators which have an $H^{\infty}$ functional calculus, in Miniconference on operator theory and partial differential equations, Proc. of CMA, Canberra, vol. 14, 1986, pp. 210-231. 
[19] McIntosh (A.) \& YAgi (A.) - Operators of type $\omega$ without a bounded $H^{\infty}$ functional calculus, in Miniconference on operators in analysis, Proc. of CMA, Canberra, vol. 24, 1989, pp. 159-172.

[20] Partington (J.R.) \& Weiss (G.) - Admissible observation operators for the right shift semigroup, Math. Cont. Signals Systems, t. 13 (2000), pp. 179-192.

[21] Weis (L.) - A new approach to maximal $L_{p}$-regularity, in Proc. of the Sixth International Conference on Evolution Equations and their Applications in Physical and Life Sciences (Bad Herrenalb, 1998) (Lumer (G.) \& Weis (L.), eds.), Lecture Notes in Pure and Appl. Math., vol. 215, Marcel Dekker, New-York, 2001, pp. 195-214.

[22] _ Operator valued Fourier multiplier theorems and maximal regularity, Math. Ann., t. 319 (2001), pp. 735-758.

[23] WeIss (G.) - Admissibility of unbounded control operators, SIAM J. Control Optim., t. 27 (1989), pp. 527-545.

[24] _ Admissible observation operators for linear semigroups, Israel J. Math., t. 65 (1989), pp. 17-43.

[25] - Two conjectures on the admissibility of control operators, in Estimation and control of distributed parameter systems, Birkäuser Verlag, 1991, pp. 367-378.

Note added on proofs. - We learned that some of the results in Section 3 were obtained independently by Bernhard Haak (Karlsruhe). His work should appear soon in his Ph.D. thesis. 